\title{
Evaluation of Visual Proprioceptive Skill in South Indian Children with Developmental Coordination Disorder
}

\author{
Ganapathy Sankar $\mathrm{U}^{1}$ and Monisha $\mathrm{R}^{2 *}$ \\ ${ }^{1} S R M$ College of Occupational Therapy, India \\ ${ }^{2}$ SRM College of Physiotherapy, India \\ *Corresponding author: Monisha R, Assistant Professor, Chennai, India
}

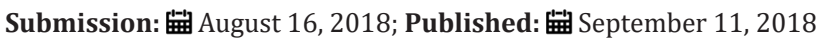

\begin{abstract}
Introduction: DCD can result from a combination of impairments in proprioception, programming of motor events and timing or in sequencing events. There has been much difficulty in identifying children with DCD because of the overlap of the child's clinical presentation with learning disability, ADHD and DAMP. Inspite of having IQ greater than 70, children with DCD when arrive in school finds reading to be an impossible task.

Objective: This study was conducted to explore the visual-proprioceptive mapping ability of children with DCD with control group study children.

Method: 15 children with the age group of 6 years referred for their motor problems in home and in school to a rehabilitation clinic. Children with Diagnosed case of any pervasive developmental disorder is excluded, included children shows IQ score of above 70 on WISC-R-intelligence test, DTVP, TPT and MPT was tested in the children with DCD and in control group.
\end{abstract}

Result: Children with DCD show significant poor performance in the entire sub test when compared with the control group.

Conclusion: Children with DCD appears to have problem in hand eye coordination and visual closure, motor component is present in all perceptual task, hence the study concludes that difficulty in motor programming contribute to visual-perceptual deficit.

Keywords: Developmental coordination disorder; Developmental test of visual proprioception; TPT; MPT; DAMP

\section{Background}

Over the years, we have seen a sharp growth in literatures and research studies conducted in children with Developmental coordination disorder (DCD). But there has been much debate on the etiology, prevalence, assessment and identification of DCD group of children's in south India [1].

This debate has widened the area of research and heralded a new revolution. Significant proportion of children with DCD presents with impaired body-eye coordination and poor acquisition of motor skills [2]. This study was conducted to explore the visualproprioceptive mapping ability of children with DCD and the control group study subjects were selected by matching every second child in the experimental group on the basis of age and gender [3]. Majority of literature search has identified that children's difficulty in coordination is because of damage to specific group of neuron or brain regions. DCD can result from a combination of impairments in proprioception, programming of motor events and timing or in sequencing events. The problems experienced by children's with
DCD are believed to be originating from defects in neuro transmitter or in the receptor system [4].

The children with DCD in this research trial revealed a significantly poorer performance than control children in visuoproprioceptive matching tasks [5]. They had poorer coordination on cross modal judgements; here visual guidance is in need to accomplish the proprioceptive perception of limb position, children in the experimental group seemed to have particular difficulty in cross model judgment [6]. Proprioception provides feedback about the relative position of body part and it helps for drawing a distinction between performance and sequencing of complex movements, the proprioceptors which carry afferent information, proprioceptive judgment of limb position can be achieved purely through sensory matching and body-centered spatial judgments posits difficulty for children with DCD [7].

Prevalence estimate of DCD is higher among boys than in girls because the behavior of boys with coordination disorder 
will be more difficult to handle at home and in school. During the early school years, children (approximately 8\%) present with coordination disorder displays poor perceptual-motor skills. There has been much difficulty in identifying children with DCD because of the overlap of the child's clinical presentation with learning disability, ADHD and DAMP. Inspite of having IQ greater than 70, children with DCD when arrive in school finds reading to be an impossible task [8]. For a child to be diagnosed as DCD, motor impairment should negatively affects the child's activity of daily living and the child should not be a diagnosed case of pervasive developmental disorder. These group of children will have no physical disability but tasks such as handwriting, catching a ball and introspecting objects will be difficult for them [9].

Continuum of research progress has been made in the development of suitable diagnostic tests and assessment tool for the identification of DCD, but unfortunately relatively little progress has been made in the development of appropriate diagnostic test and understanding the deficits in perceptual-motor function in these children is still under investigation [10]. Lack of classical signs of neurological damage and physical disability, understanding the underlying factors for the visual perceptual deficit is particularly difficult and highly disappointing. Till date there has been no appropriate therapy to improve their coordination and associated co-morbidities [11]

Proprioception is body centered, information from the proprioceptors needs to be correlated with visual information about the position of limb in relation to the object to be moved. Visual cues are needed to locate and identify the appropriate object to be moved [12]. In postulating the perceptual skills, deficits in vision and visual processing contribute to the problems of children with DCD and this have been proposed to contribute to the 'clumsiness'.

Previous research has identified perceptual processing deficit in children with DCD, but the performance of individual children's proprioceptive skill has not been highlighted [13]. Hence this study have been done to find the individual difference in visualproprioceptive skill acquisition and the difficulties faced by the children in timing and sequencing the motor task in DCD.

\section{Method}

15 children referred for their motor problems in home and in school to a rehabilitation clinic. Children with the age group of 6 years and not a diagnosed case of any pervasive developmental disorder and didn't have any indicated neurological deficit or physical impairment. IQ score of above 70 on WISC-R- intelligence test. Out of 15, 10 children scored greater than 70 on intelligence test were included in this study. Movement assessment battery for children $(A B C)$ was administered to the study sample and 9 children have impairment score below $5^{\text {th }}$ percentile and they are the definite case of DCD with predominant motor problem and the remaining 1 child have completed MABC didn't shows impairment under motor domain, hence excluded out of the study. 9 children also had a poor performance on 4 checklists. Therefore a total of 9 had participated in this study. To find the difference in these 9 children for their dominant hand performance, Pen and paper was placed before all these 9 children and they were instructed to write their names to identify the dominant limb for initiating movement.

\section{Procedure}

Children with DCD and the control group children were assessed in the quite room with MABC and in addition manual pointing task was administered to all the children in the study. In Test number one, Children were asked to discriminate the reversal and rotation of numbers and to identify the number as per instruction in order to test the visual perception. In test two, they were asked to find out the number which is hidden by lines. In test three, children were asked to complete the incompletely drawn lines and to find out the resultant numbers and figures and in test four; children were tested with their ability to match two figures or numbers with different colors and presented with different size [14]. These four test measures the visual perception of all the 9 children presented with DCD. Another four test has been administered to document the visual motor integration. First the ability of child to draw a straight line and to follow the curved figure has been tested. In second test of motor integration they were asked to copy down the figure or number which has been be drawn at the side of the paper [15]. Third test was on spatial relation here the child has been asked to connect all the dots in orderly fashion and to find out the resultant figure or number, final test was on visual motor speed, they were asked to draw small and large cubes inside the fixed dimension and the time for completion of the task has been documented to analyze the speed. This Developmental Test of visual perception administered to all the 9 participants and compared with the control group participants. Raw score for these 8 subtest has been calculated and computation has been made for the total score to know the child's general visual perception (GVP). By administering the DTVP- 2, motor reduced visual perception and visual motor integration has been analyzed for these 9 children in DCD group. Tactile perception of these children has been tested by using geometric figures and boards, by instructing the child to match the appropriate shape of cubes and figures in the specified boxes and to measure the tactile perception, here the child vision has been occluded and by active manipulation of object, the child have to complete the task and the time taken to fill in the boxes with appropriate shapes with dominant and non-dominant hand has been recorded [16]. Number of boxes corrected matched with appropriate figures has been documented. This test has been done to analyze the hepatic perception. Raw score of this Tactual performance test has been calculated and converted into t score. Manual pointing task has been conducted to all the participants, here the child has been asked to plot the bulletin pin at the prescribed location set by the experimenter on the A4 paper. Proprioception has been tested here by asking the participant to locate the dot marked by the experimenter by the non dominant hand of the child and then the vision has been occluded to plot the bulletin pin at the exact location with the dominant hand [17]. The corners of the paper have been fixed in the table with 4 pins and four trials has been conducted the placement error in $\mathrm{x}$ and $\mathrm{y}$ axis has been documented with the four corner pins as reference point. The distance between the misplaced bulletin pin has been calculated in relation to the reference point [18] 


\section{Data Analysis}

Table 1-3.

Table 1: Number of children with DCD and control group on developmental test of visual perception (DTVP-2) scored below the $15^{\text {th }}$ percentile.

\begin{tabular}{|c|c|c|c|}
\hline S.NO & Sub-Test & $\begin{array}{c}\text { Children } \\
\text { With DCD }\end{array}$ & $\begin{array}{c}\text { Control Group } \\
\text { Children }\end{array}$ \\
\hline & Eye-hand co-ordination & 8 & 0 \\
\hline & copying & 7 & 0 \\
\hline & Spatial relation & 5 & 1 \\
\hline & Visual motor speed & 7 & 1 \\
\hline & Position in space & 6 & 0 \\
\hline & Figure ground & 6 & 0 \\
\hline & Visual closure & 9 & 2 \\
\hline & Form constancy & 9 & 2 \\
\hline & MRP & 8 & 1 \\
\hline & VMI & 7 & 1 \\
\hline
\end{tabular}

Table 2: Number of children with DCD and control children on TPT below $15^{\text {th }}$ percentile - total score less than 36 .

\begin{tabular}{|c|c|c|c|}
\hline S.NO & Condition & DCD & Control \\
\hline & Preferred hand & 3 & 0 \\
\hline
\end{tabular}

\begin{tabular}{|c|c|c|c|}
\hline & Non preferred hand & 4 & 1 \\
\hline & Both hands & 5 & 1 \\
\hline & memory & 1 & 0 \\
\hline
\end{tabular}

Table 3: Manual pointing task- (standard error) Mean and SD between DCD and control subjects.

\begin{tabular}{|c|c|c|c|c|c|}
\hline \multirow{2}{*}{ S.NO } & \multirow{2}{*}{ Condition } & \multicolumn{2}{|c|}{ ChildDED with } & \multicolumn{2}{c|}{ Control } \\
\cline { 3 - 6 } & & Mean & SD & Mean & SD \\
\hline & Dominant hand- Visual & 3.1 & 2 & 1.8 & 0.05 \\
\hline & Visual proprioceptive & 2.5 & 1.7 & 1.5 & 0.9 \\
\hline & proprioception & 2.7 & 1.9 & 1.8 & 0.05 \\
\hline & Non-dominant hand-Visual & 3.7 & 2.4 & 2 & 0.46 \\
\hline & Visual proprioceptive & 3 & 2 & 2 & 0.46 \\
\hline & proprioception & 3.7 & 2.4 & 1.7 & 0.04 \\
\hline
\end{tabular}

\section{Result}

Children with DCD show significant poor performance in the entire sub test when compared with the control group. Children with DCD appears to have problem in hand eye coordination and visual closure, significant difference has been obtained in children with DCD and control group for other sub test copying, spatial relation, visual motor speed, position in space (Figure 1-3).

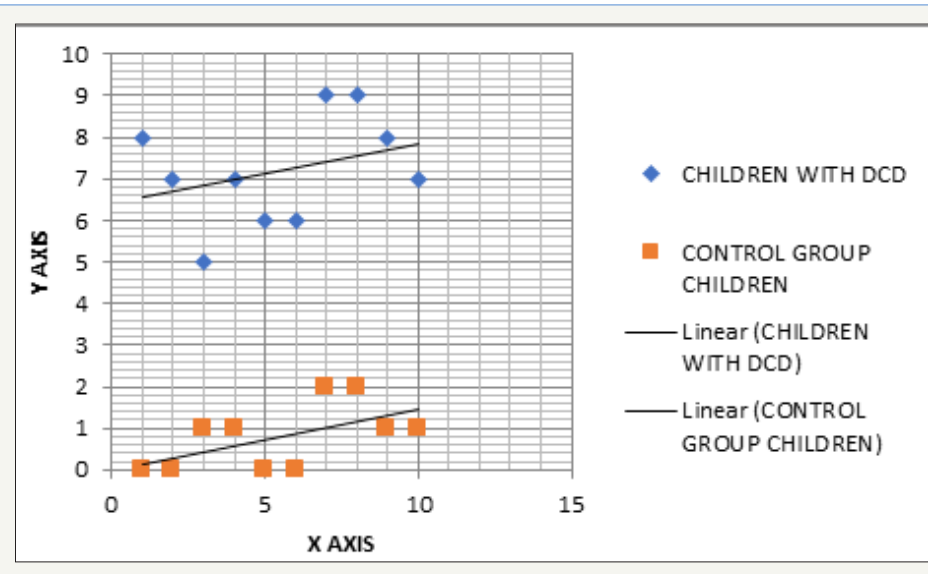

Figure 1: Graph for Table 1.

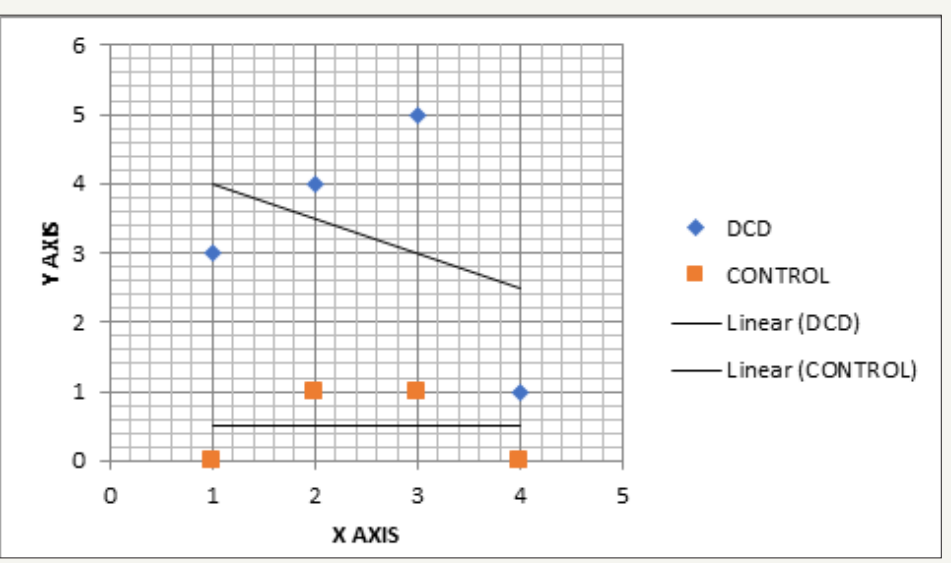

Figure 2: Graph for Table 2. 


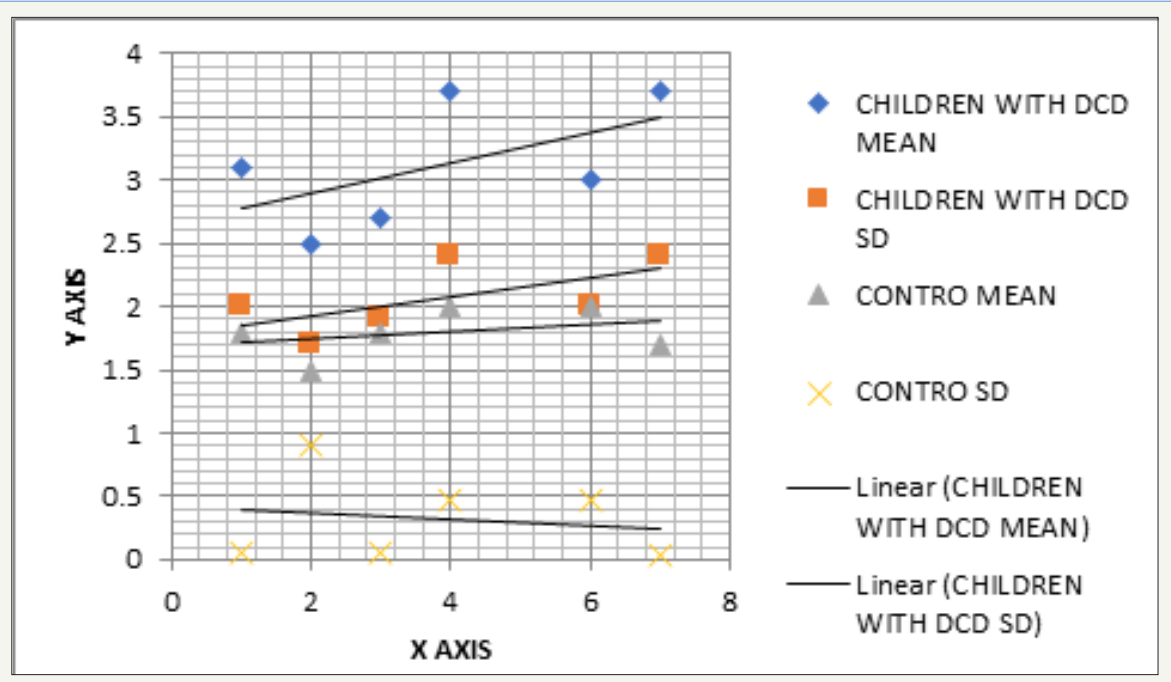

Figure 3: Graph for Table 3..

\section{Discussion}

This study aims to analyze whether children with DCD have problem with visual proprioceptive information. Wilson and McKenzie (2001), in previous studies, general isolated aspects of visual, proprioceptive or tactile skills were investigated. Children with DCD have difficulty in detecting figures that were hidden in a complicated background and they show poor performance in matching figures that vary in size and in color. They also had difficulty in discriminating pairs of triangle with same or different size. Holmen (2000) in his research work in DCD had identified visual spatial impairments for children with DCD in joining the presented straight lines or dotes. Moran (1992) in his study on visual-spatial impairments concluded the study with conflicting results and the results are difficult to explain, when the methodology of his research work has reviewed it shows that, the sample he selected in trial were from age group of 5 to 15 and he have highlighted that the inclusion criteria is not responsible for this conflicting result. Children with DCD find it difficult to recognize figure that is incompletely drawn [19].

Overall, the problems of children with DCD become most pronounced on vasuomotor subtests of DTVP-2. They have poor eye- hand coordination to draw between lines, to copy a figure, to connect dots, and to complete the particular drawing task in time. Spatial relations, visual-motor speed subtest is highly difficult in children with DCD.

This high percentage of difficulty in achieving visual motor skill warrant clinical attention. Van Dellen in his research work has provided the group difference in perceptual skills, without providing information on the individual perceptual deficit. The present study has shown the individual perceptual deficit with the statistical mean value and SD value.

Previous research has highlighted the causal relationship between DCD and deficit in visual perception. But no study had done analyzing the relationship between severity of DCD identified by MABC and proprioceptive skill, lot of research trials failed to identify the cause and the relationship between perceptual skill and motor coordination disorder. Therefore, the findings of this study has identified that children with DCD have problems in visual, proprioceptive and tactile skills [20].

\section{Conclusion}

Children with DCD appears to have problem in hand eye coordination and visual closure, motor component is present in all perceptual task, hence the study concludes that difficulty in motor programming contribute to visual-perceptual deficit.

\section{References}

1. American Psychiatric Association (1987) Diagnostic and statistical manual of mental disorders. ( $3^{\text {rd }}$ edn), Washington DC, USA.

2. American Psychiatric Association, Bairstow PJ, Laszlo JI (1981) Kinaesthetic sensitivity to passive movements and its relationship to motor development and motor control. Developmental Medicine and Child Neurology 23: 606-166.

3. Barnett A, Henderson SE (1992) Some observations on the figure drawings of clumsy children. British Journal of Educational Psychology 62: 341-355.

4. Doyle AJR, Elliott JM, Connolly KJ (1986) Measurement of kinaesthetic sensitivity. Developmental Medicine and Child Neurology 28(2): 188193.

5. Dwyer C, McKenzie BE (1994) Impairment of visual memory in children who are clumsy. Applied Physical Activity Quarterly 11: 179-189.

6. Elliott JM, Connolly KJ, Doyle AJR (1988) Development of kinaesthetic sensitivity and motor performance in children. Developmental Medicine and Child Neurology 30(1): 80-92.

7. Field J (1976) Relation of infants reaching behaviour to stimulus distance and solidity. Developmental Psychology 5: 444-448.

8. Graham M, Levene MI, Trounce JQ, Rutter N (1987) Prediction of cerebral palsy in very low birthweight infants: prospective ultrasound study. Lancet 2(8559): 593-536.

9. Sugden DA (1992) Movement assessment battery for children London: The psychological corporation, Harcourt BraceJovanovich.

10. Hoare D (1994) Subtypes of developmental coordination disorder. Applied Physical Activity Quarterly 11: 158-169. 
11. Larkin D (1991) Kinaesthetic abilities of clumsy children. Dev Med Child Neurol 33(8): 671-678.

12. Hulme C, Biggerstaff A, Moran G, McKinley I (1982a) Visual, kinaesthetic and cross-modal judgements of length by normal and clumsy children. Dev Medi Child Neurol 24(4): 461-471.

13. Smart A, Moran G (1982b) Visual perceptual deficits in clumsy children Neuropsychologia 20(4): 475-481.

14. Huynh H, Feldt LS (1970) Conditions under which mean square ratios in repeated measure designs have exact F-distributions. Journal of the American Statistical Association 65(332): 1582-1589.

15. Jongmans M, Henderson S, de Vries L, Dubowitz L (1993) Duration of periventricular densities in preterm infants and neurological outcome at 6 years of age. Arch Dis Child 69: 9-13.

16. Laszlo JI, Bairstow PJ (1983) Kinaesthesia: its measurement, training and relationship to motor control. Quarterly Journal of Experimental Psychology 35: 411-421.

17. Mon-Williams MA, Pascal E, Win JP (1994) Ophthalmic factors in developmental coordination disorder. Applied Physical Activity Quarterly 11(2): 170-178.

18. Conway R, McCulloch DL, Pascal E (1996) Visual evoked potentials in children with developmental coordination disorder. Ophthalmic and Physiological Optics 16(2): 178-183.

19. Mutch L, Leyland A, McGee A (1993) Patterns of neuropsychological function in a low birthweight population. Developmental Medicine and Child Neurology 35(11): 943-956.

20. Polatajko HJ, Macnab JJ, Anstett B, Malloy-Miller T, Murphy K, et al. (1995) A clinical trial of the process-oriented treatment approach for children with developmental coordination disorder. Dev Med Child Neurol 37(4): 310-319.

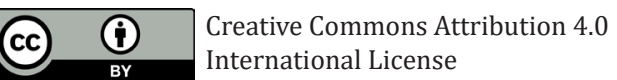

For possible submissions Click Here 